\title{
Glucose-Insulin Pharmacodynamic Surface Modeling Comparison
}

\author{
J. Geoffrey Chase*, Steen Andreassen.**, Ulrike Pielmeier**, Christopher E. Hann* \\ *Mechanical Eng, Centre for Bio-Engineering, University of Canterbury, Christchurch, New Zealand \\ (Tel: +64-3-364-7001; e-mail: geoff.chase@ Canterbury.ac.nz). \\ **Center for Model-based Medical Decision Support (MMDS), Aalborg University, Aalborg, Denmark (e-mail: \\ sa@hst.aau.dk)
}

\begin{abstract}
Metabolic system modeling for use in glycaemic control is increasing in importance. Few models are clinically validated for both fit and prediction ability. For such models, this research introduces a new form of pharmaco-dynamic (PD) surface comparison for model validation. These 3D surfaces are developed for 3 validated models, including the well-known Minimal Model, and fit to clinical data. The approach is clearly highlights differences in modeling methods, dynamics utilized and physiological assumptions that may not appear as clearly in other validation approaches. The deficiencies of the Minimal Model in comparison to more physiologically representative models are illustrated in this context.
\end{abstract}

\section{INTRODUCTION}

Type 1 and Type 2 diabetes are epidemic with significant economic cost driven by the inability of individuals, and their clinicians, to adequately control blood glucose levels. Hence, the rate of costly chronic complications is rising. Critical care studies (van den Berghe et al (2001); Krinsley (2002)) have shown tight glycaemic control can significantly reduce mortality and cost (Krinsley, 2006). However, achieving it has proven difficult, even though initial results show tighter control is better (Chase et al, 2007; Egi et al, 2007).

The potential of models for managing glycaemic levels in any insulin resistant cohort is thus of growing import. However, relatively few models have been clinically validated. For most, the primary form of validation has been simple fitting of the model to clinical data. Occasionally, more rigorous prediction validation is used. However, only a few clinically validated models can predict within clinically acceptable ranges (e.g. Wong et al, 2006; Lotz et al, 2006; Arleth et al, 2000; Hovorka et al, 2004).

This paper presents a new form of model validation, by examining the steady state pharmaco-dynamic (PD) surfaces, including underlying pharmaco-kinetics (PK). A 3D surface of plasma insulin (x), plasma glucose (y) and resulting rate of change in endogenous glucose balance $(\mathrm{z})$ is compared to 77 sets of glycaemic clamp data (Arleth et al, 2000), creating a new form of clinical model validation in the comparison.

\section{METHODS}

\subsection{Models}

Three clinically validated models are used in this analysis:

1. Non-linear PK/PD Model (ND)

2. Minimal Model (MM)

\section{Receptor Model (RM)}

The ND model has been used in several critical care studies (e.g. Wong et al, 2006). The MM is well documented and used (Pacini et al, 1986). The RM model was developed for a Type 1 diabetes decision support system (Arleth et al, 2000).

The dynamic models (MM, ND) share the same basic PD formulation and can be jointly defined (Lotz et al, 2006):

$$
\dot{G}=-p_{G} G-S_{I} \frac{G}{1+\alpha_{G 2} G} \frac{Q}{1+\alpha_{G} Q}+\frac{P(t)}{V_{G}}+\frac{P_{\text {end }}}{V_{G}}
$$

where $G(t)[\mathrm{mmol} / \mathrm{L}]$ is the total plasma glucose, $Q(t)[\mathrm{mU} / \mathrm{L}]$ is interstitial insulin assumed equal to plasma insulin in this steady state analysis, $P(t)[\mathrm{mmol} / \mathrm{min}]$ is exogenous carbohydrate appearance, $P_{\text {end }}[\mathrm{mmol} / \mathrm{min}]$ represents endogenous glucose appearance in a glucose distribution volume $V_{G}$. Patient endogenous glucose clearance and insulin sensitivity are $p_{G}[1 / \mathrm{min}]$ and $S_{I}$ [L/(mU.min)]. MichaelisMenten function $\alpha_{G}[\mathrm{~L} / \mathrm{mU}]$ captures saturation of plasma insulin disappearance and glucose uptake by insulin, and $\alpha_{G 2}$ [L/mmol] allows for the saturation of glucose-dependent glucose clearance.

Clinical trials, fitting and prediction validation, and model details for the ND model can be found elsewhere (e.g. Wong et al, 2006; Hann et al, 2005). The Minimal Model (MM) is thus effectively the case where: $P_{\text {end }}=\alpha_{G 2}=\alpha_{G}=0,-p_{G} * G$ is redefined as $-p_{G^{*}}\left(G-G_{E}\right)$, where $G_{E}$ is a basal glucose value not used in the ND model of Equation (1), but defined in the MM (e.g. Pacini et al, 1986), and $S_{I}$ is the ratio used in the original MM formulation for active insulin.

In this steady state PD surface analysis, $Q(t) \rightarrow Q_{s \mathrm{~s}}$ and $G(t)$ $\rightarrow G_{s s}$, and $\dot{G} \Leftrightarrow P_{s s}$ in Equation (1) represent the endogenous balance change for that input in the steady state. Hence, the 
steady state ND and MM model pharmaco-dynamics can be obtained from versions of Equations (1).

The receptor model (RM) is defined (Arleth et al, 2000):

$$
\begin{aligned}
& \text { EndoBal }=\text { Hepatic }- \text { Renal }- \text { P13 }- \text { P14 } \\
& \text { Hepatic }=-0.46 \mathrm{~min}^{-1} G-1.475 \mathrm{mmol} / \mathrm{min} / \mathrm{mU} I_{s}+ \\
& 1.259 \mathrm{mmol} / \mathrm{L} / \mathrm{min} ; \quad G_{\max }=12.0 \mathrm{mmol} / \mathrm{L} \\
& \text { Renal }=0.004 \mathrm{~L} / \mathrm{mmol} / \mathrm{min} G^{2}-0.064 \mathrm{~min}^{-1} G+ \\
& 0.278 \mathrm{mmol} / \mathrm{L} / \mathrm{min} \\
& \mathbf{P 1 3}=0.56 \mathrm{~min}^{-1} * G /(G+1.5 \mathrm{mmol} / \mathrm{L}) \\
& \mathbf{P 1 4}=5.09 \mathrm{mmol}^{-} \mathrm{L} / \mathrm{min} / \mathrm{mU} G^{*} I_{s} /(G+5.0 \mathrm{mmol} / \mathrm{L}) \\
& I_{s}=I^{*} 1 /\left((I-0.083 \mathrm{mU} / \mathrm{L})^{1.77}+(0.539 \mathrm{mU} / \mathrm{L})^{1.77}\right)^{(1 / 1.77)}
\end{aligned}
$$

where all terms and values are as defined in Arleth et al, (2000). Note that this model is defined based on clinically observed gains/losses from the liver (Hepatic), and losses to the kidneys (Renal), insulin-dependent periphery (P14) and insulin-independent periphery (P13). Hence, this receptor based model is physiologically defined. Finally, $I_{s}$ is a saturated insulin effect for insulin-dependent uptake, and it should be noted that P13 is a saturable insulin independent uptake of glucose and P14 a saturable insulin dependent uptake. The other specific values are obtained by fitting to 77 clinical studies.

The net results of this model (EndoBal) creates the 3D PD surface used for this comparison. This surface must be mathematically defined for the MM and ND models. Note that the RM model was created by directly fitting to clinical data, and thus needs no further alteration or analysis.

\subsection{PD Surface Fitting and Error Metrics}

To fit the PD surface for the ND and MM cases, a grid search was used over physiological ranges of important variables. In this case, the values of $\alpha_{G 2}, \alpha_{G}, S_{I}$ were allowed to vary with $P_{\text {end }}, P_{G}$, and $V_{G}$ set to constants, as in Table 1. The physiological ranges for $\alpha_{G 2}, \alpha_{G}, S_{I}$ are based on an extensive literature search, model validation study and sensitivity study (Hann et al, 2005), and are also shown in Table 1 for normal subjects, as the clinical studies utilised normal subjects.

Table 1: Variable values used in ND and MM analyses

\begin{tabular}{|l|lr|}
\hline & \multicolumn{1}{|l|}{ Value } \\
\hline \hline$P_{G}$ & \multicolumn{1}{|l|}{$0.006 \mathrm{~min}^{-1}$} \\
\hline$V_{G}$ & $13.3 \mathrm{~L}($ for $70 \mathrm{~kg}), V_{G}=0.19 *$ Mass $(\mathrm{kg})$ \\
\hline$P_{\text {end }}$ & $1.2 \mathrm{mmol} / \mathrm{L} / \mathrm{min}$ & (0 for the MM case) \\
\hline \hline$S_{I}$ & $1.5 \mathrm{e}-3-3.5 \mathrm{e}-3 \mathrm{~L} / \mathrm{mU} / \mathrm{min}$ \\
\hline$\alpha_{G}$ & $1 / 20-1 / 80 \mathrm{~L} / \mathrm{mU}$ & (0 for the MM case) \\
\hline$\alpha_{G 2}$ & $1 / 5-1 / 20 \mathrm{~L} / \mathrm{mmol}$ & (0 for the MM case) \\
\hline
\end{tabular}

For each set of variables in the lower part of Table $1, \dot{G}=-P_{s s}$ is calculated from the model at the $\left(Q_{s s}, G_{s s}\right)$ from each of the 77 clamp studies, creating 77 sets of $\left(Q_{s s}, G_{s s}\right) \rightarrow \dot{G}=-P_{s s}$ points for each combination of $\left(\alpha_{G 2}, \alpha_{G}, S_{I}\right)$. Importantly, this approach assumes that the steady state glucose infusion in the clamp $\left(P_{s s}\right)$ is the negative of the glucose rate of change $(\dot{G})$ that would occur without it. Hence, these terms represent the same value, with $P_{s s}$ for the clamp infusion to maintain euglycaemia and $\dot{G}$ for the equivalent endogenous balance change that would occur for the given glucose and insulin levels without it. The difference is in the sign with $P_{s s}$ positive and $\dot{G}$ negative in this usage.

Details on the clamp cohort $(\mathrm{n}=77)$ are in Arleth et al (2000) but consist of a wide selection of euglycaemic and hyperglycaemic clamp studies in the literature.

Overall, 11,424 variable sets $\left(\alpha_{G 2}, \alpha_{G}, S_{I}\right)$ result in 77 error values (per set) in endogenous balance, summarised by:

1. RMS Error (RMS) - this metric rewards solutions that minimise error over all studies.

2. Absolute Mode of Error (AME) - most frequent absolute error, rewarding solutions with more errors near zero and allows more outliers to account for variation observed in clamp studies.

3. Frequency of Error Near Zero (FNZ) - number of errors within $+/-0.025$ of 0.000 error value or $\sim 5 \%$ of mean $\dot{G}$ observed in the clinical studies. This metric rewards solutions that have greater concentrations within observed glycaemic clamp variability of 5\%, regardless of the specific mode.

Each error metric rewards different quality of fit. RMS minimises outliers, without accounting for outlying clinical results or variation. AME rewards solutions that maximise "inliers" but not to a specific value. FNZ rewards "inlier" solutions within clinically observed variability for glycaemic clamps, allowing outliers for study variability and error. The grid search results were analysed based on all three metrics, and compared to the RM results. Differences are then discussed in the context of the model dynamics.

Note that the RM model of Equations (2)-(7) was created by direct fitting to this data, and needs no further alteration.

\section{RESULTS \& DISCUSSION}

Results are presented for 2 basic model types from Equations (1)-(3). The MM model where there is no saturation $\left(\alpha_{G}=\alpha_{G 2}\right.$ $=0)$, and the ND model with either 1 non-zero saturation $\left(\alpha_{G}\right)$ term denoted ND1, or with both saturation terms $\left(\alpha_{G}\right.$ and $\left.\alpha_{G 2}\right)$, which is denoted ND2.

Figure 1 shows the histograms comparing the ND2 model when sorted by RMS and FNZ. FNZ and AME results were similar, so the latter is not shown. FNZ allows more outliers, but has greater numbers around zero. Qualitatively FNZ was used because the results were almost identical in the ND2 case, and identical in the ND1 and MM cases. The use of FNZ may account slightly more for variation in methods and procedures in the clinical study than RMS.

Figure 2 compares the errors for the ND1 and RM cases. As expected, the RM model is tighter having been fit to this clinical data in its derivation. The ND2 model error is more tightly distributed than ND1. Interestingly, the ND1 and ND2 models had greater FNZ than the RM model, but slightly 
wider distributions. The MM is not shown due to its very wide distribution of errors.

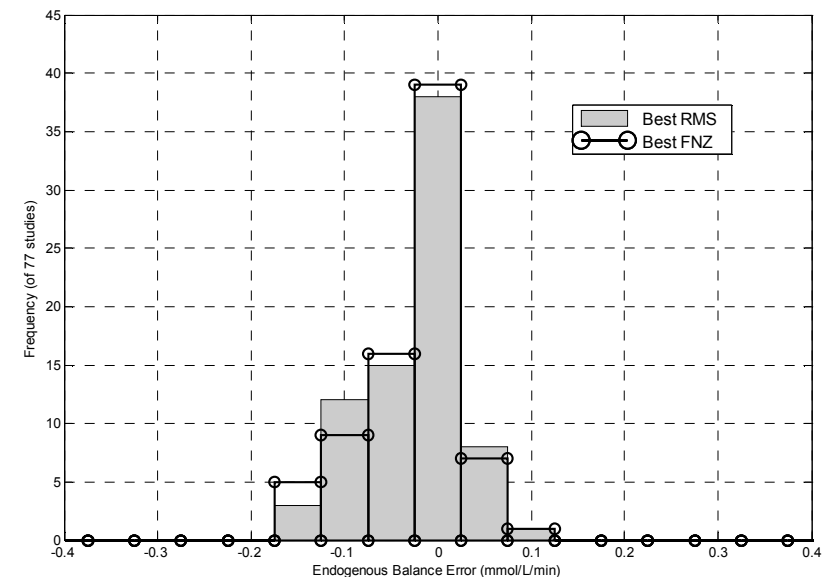

Fig. 1: ND2 error histogram, RMS vs FNZ metrics

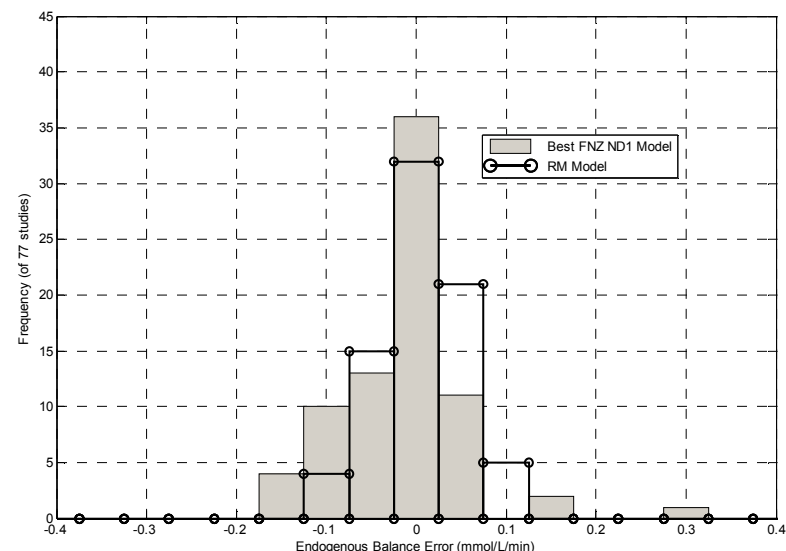

Fig. 2: ND1 and RM errors. Note ND1 results were identical for RMS and FNZ metrics.

FNZ sorted error results are given in Table 2, with relatively tight ND1 and ND2 distributions, compared to the RM model. In contrast, the MM has very large error and outliers.

Table2: Results (parameter values and errors). Best FNZ and $\mathrm{RMS}$ results are identical for the ND1, MM and RM

\begin{tabular}{|l|l|}
\hline Model & Values \\
\hline \hline ND1 & $\alpha_{\mathrm{G}}=1 / 48 \mathrm{~L} / \mathrm{mU}, \alpha_{\mathrm{G} 2}=0 \mathrm{~L} / \mathrm{mmol}$, \\
& $\mathrm{S}_{\mathrm{I}}=0.0016 \mathrm{~L} / \mathrm{mU} / \mathrm{min}$ \\
& $\mathrm{RMS}=0.07 ; \mathrm{AME}=-0.01, \mathrm{FNZ}=36$ \\
\hline ND2 & $\alpha_{\mathrm{G}}=1 / 44 \mathrm{~L} / \mathrm{mU}, \alpha_{\mathrm{G} 2}=1 / 7 \mathrm{~L} / \mathrm{mmol}$, \\
Best FNZ & $\mathrm{S}_{\mathrm{I}}=0.0029 \mathrm{~L} / \mathrm{mU} / \mathrm{min}$ \\
& $\mathrm{RMS}=0.06 ; \mathrm{AME}=-0.01, \mathrm{FNZ}=39$ \\
\hline ND2 & $\alpha_{\mathrm{G}}=1 / 50 \mathrm{~L} / \mathrm{mU}, \alpha_{\mathrm{G} 2}=1 / 5 \mathrm{~L} / \mathrm{mmol}$, \\
Best RMS & $\mathrm{S}_{\mathrm{I}}=0.0032 \mathrm{~L} / \mathrm{mU} / \mathrm{min}$ \\
& $\mathrm{RMS}=0.05 ; \mathrm{AME}=-0.00, \mathrm{FNZ}=38$ \\
\hline MM & $\alpha_{\mathrm{G}}=\alpha_{\mathrm{G} 2}=0 \mathrm{~L} / \mathrm{mU} \& \mathrm{~L} / \mathrm{mmol}$ \\
& $\mathrm{S}_{\mathrm{I}}=0.0015 \mathrm{~L} / \mathrm{mU} / \mathrm{min}$ \\
& $\mathrm{RMS}=4.77 ; \mathrm{AME}=-0.05, \mathrm{FNZ}=3$ \\
\hline RM & $\mathrm{RMS}=0.04 ; \mathrm{AME}=-0.01, \mathrm{FNZ}=32$ \\
\hline
\end{tabular}

ND1, ND2 and RM are similar, while the MM model is quite different. Sorting for best RMS or FNZ for ND2 has no effect, and optimal solutions were robust over the variables. However, small differences in error are more evident in waterfall plots of the insulin-endogenous balance plane.

Figures 3-4 show these waterfalls for the MM. Outlying errors are clearly due to the linear nature of the MM model glucose-insulin dynamics in this plane. In these figures, lines of constant glucose increase in value from the top to bottom lines, with clinical data marked individually. The MM errors will be very large for the higher insulin level values in Figure 3 , resulting in the large RMS error of Table 2.

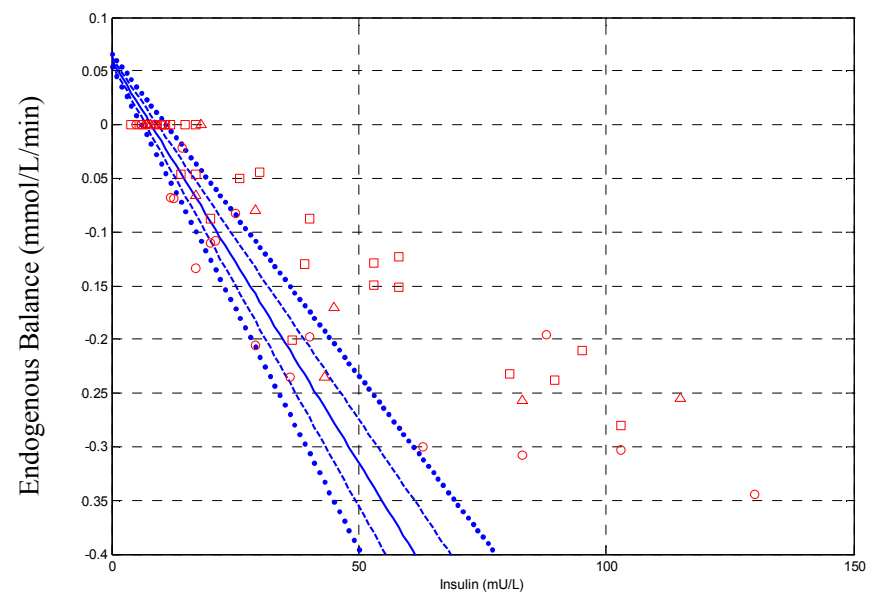

Fig. 3: MM waterfall with lines showing constant glucose at $G_{s s}=4.0,4.5,5.0,5.5$ and $6.0 \mathrm{mmol} / \mathrm{L}$. Clinical Studies: Squares: $G_{s s}=4-4.75 \mathrm{mmol} / \mathrm{L}$, Circles: $G_{s s}=4.75-5.25$ $\mathrm{mmol} / \mathrm{L}$ and Triangles: $G_{s s}=5.25-6.0 \mathrm{mmol} / \mathrm{L}$.

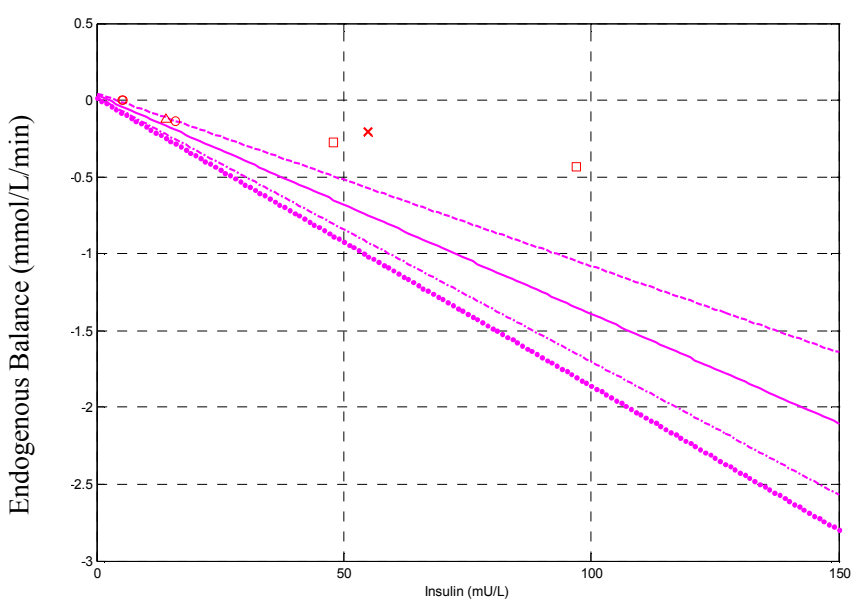

Fig. 4: MM waterfall with lines showing constant glucose at $G_{s s}=7.5,9.5,11.5,12.5 \mathrm{mmol} / \mathrm{L}$. Clinical Studies: Circles: $G_{s s}=7-8 \mathrm{mmol} / \mathrm{L}$ studies, Triangles: $G_{s s}=9-10 \mathrm{mmol} / \mathrm{L}$, Squares: $G_{s s}=10-11 \mathrm{mmol} / \mathrm{L}$, and the $\mathrm{X}$ is $12-13 \mathrm{mmol} / \mathrm{L}$.

Figures 5-8 show the waterfall plot results for the ND1 and ND2 models. The ND2 figures are for the values sorted for best FNZ results. The results of Figures 5-8 are significantly improved versus the MM results, as also seen in Table 2 . This difference is largely due to the MM model's linear 
removal of glucose as a function of insulin (at a given glucose level), which prevent it from capturing clinical values above (relatively) very low insulin values. Hence, the saturation that is clearly evident in the clinical data cannot be captured by the MM dynamics, resulting in very large error values over the entire clinical data set.

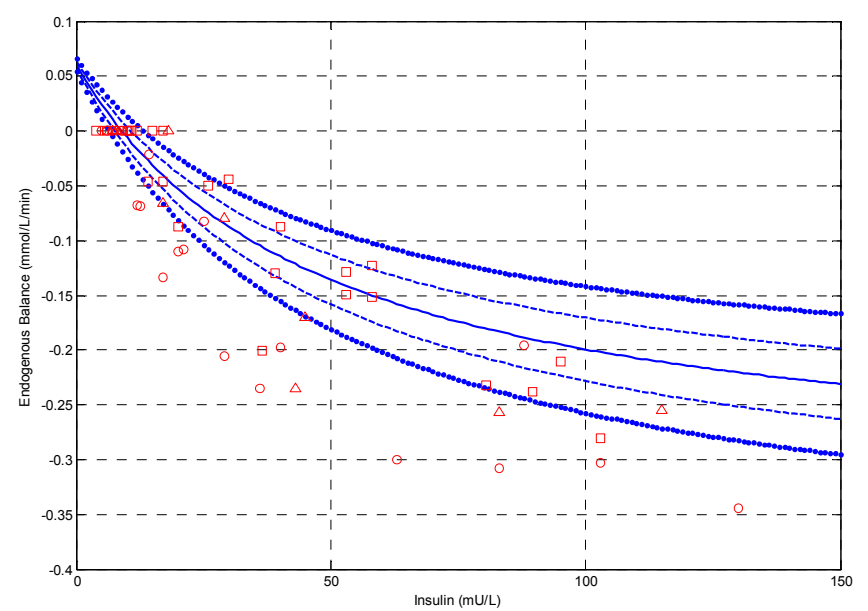

Fig. 5: ND1 waterfall with data as defined in Figure 3.

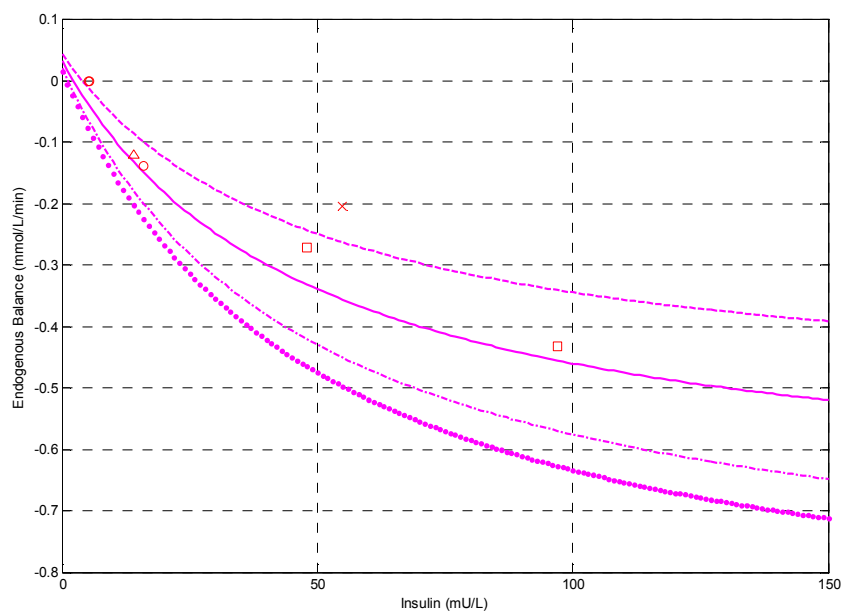

Fig. 6: ND1 waterfall with data as defined in Figure 4.

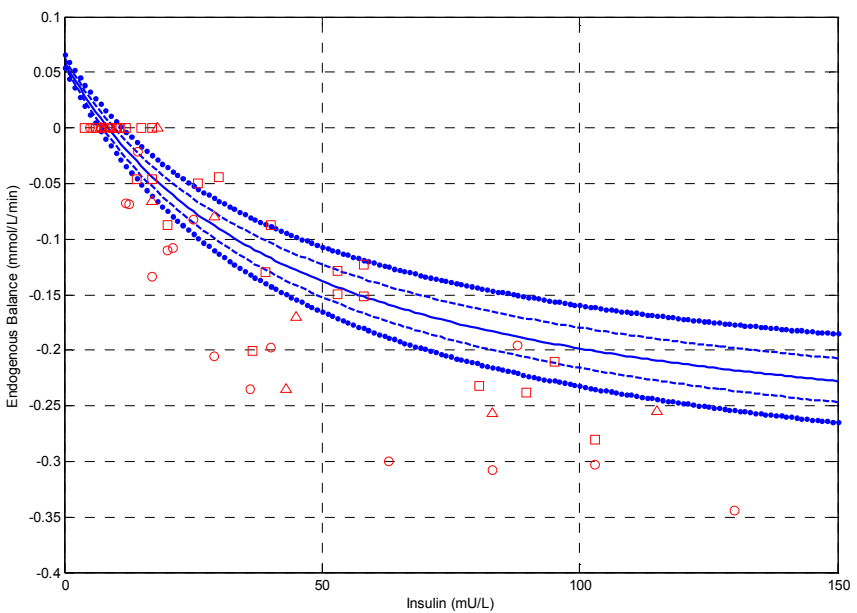

Fig. 7: ND2 waterfall with data as defined in Figure 3.

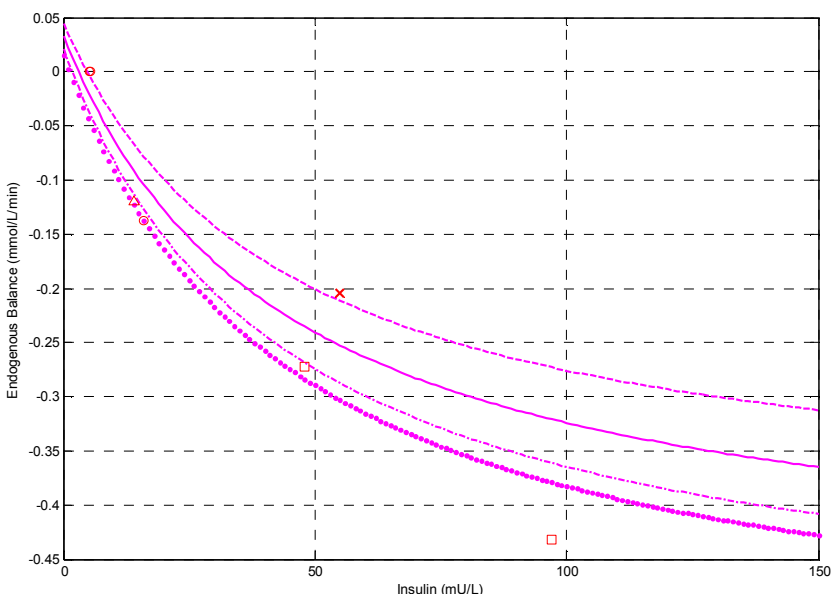

Fig.8: ND2 waterfall with data as defined in Figure 4.

However, the ND models also fail to fully capture some clinical values. However, Figures 5 and 7 in particular show that from $30-100 \mathrm{mU} / \mathrm{L}$ on the insulin axis both models have difficulty capturing some of the clinical data points.

Figures 9-10 show the RM model waterfalls. These plots more accurately fit the central portion of the clinical data, as expected due to the direct fitting of the model to this data. A salient difference is provided by the highly non-linear saturation curve of Equation (7), allowing the tighter distribution of constant glucose lines seen in these figures.

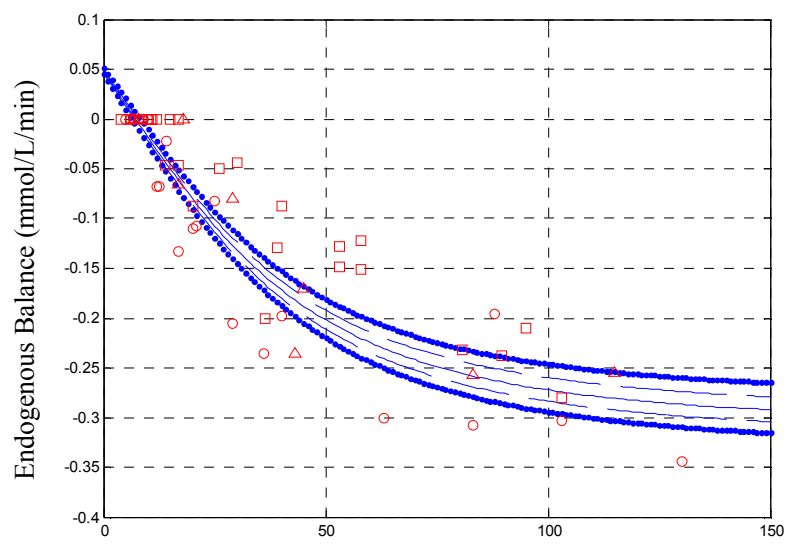

Fig.9: RM waterfall with data as defined in Figure 3.

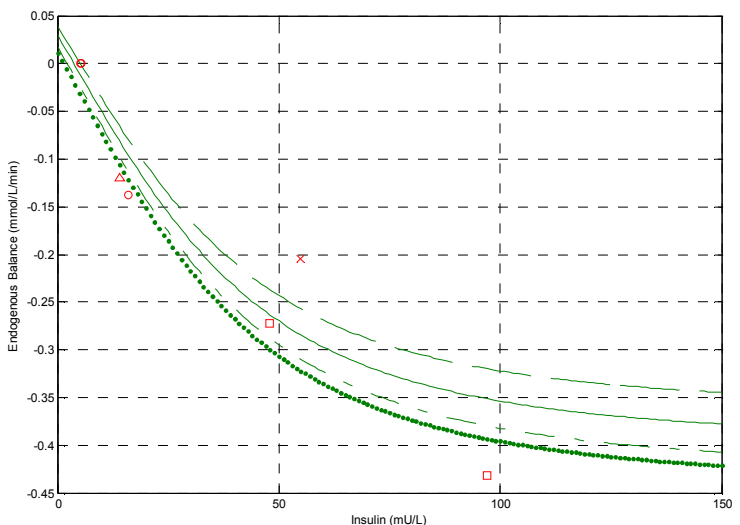

Fig.10: RM waterfall with data as defined in Figure 4. 
From the ND to RM models the difference in fit and behaviour are due primarily to two factors:

1. A highly non-linear insulin effect saturation curve fit to the clinical data (Equation (7))

2. A separate saturated glucose loss in the periphery in Equation (5), along with independent saturation of glucose and insulin in Equations (6)-(7).

The first difference appears in the insulin-endogenous balance plane and is illustrated in Figure 11. The RM model insulin saturation curve (scaled) is much sharper than the Michaelis-Menten saturation of the ND models. Different Michaelis-Menten constants thus fit the clinical data better at different insulin levels, but none work well over the entire range used here. In this case, 2-3 Michaelis-Menten constants work well in different ranges, where the RM fitted to the clinical data works over all ranges for this data.

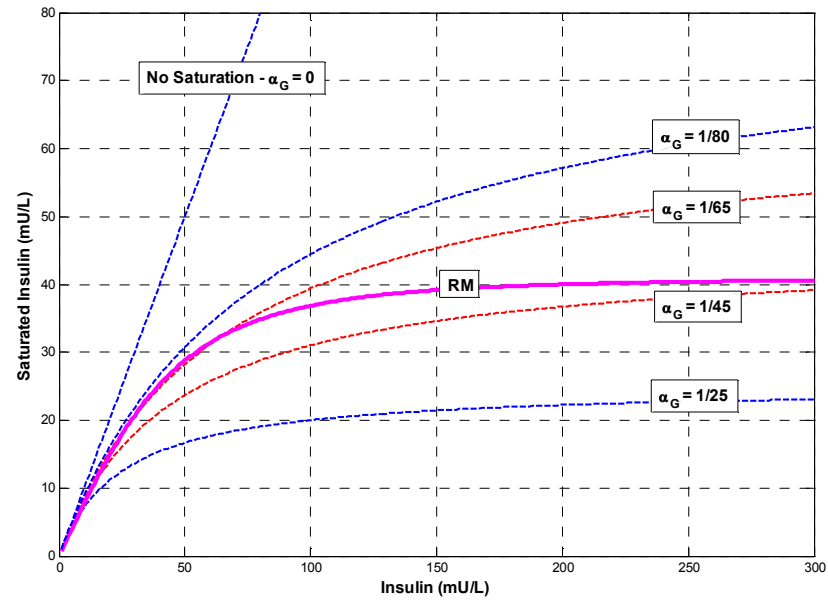

Fig 11: Comparison of insulin saturation curves

The sharper, highly non-linear saturation curve also results in the closer lines of constant glucose in Figures 9-10. The softer Michaelis-Menten term only offers the wider glucose curves in Figures 5-8. Thus, the ND model provides a lesser fit to this clinical data, which is more sharply saturated, as seen in all of Figures 3-10. Note that while the ND2 model is tighter with its second saturation, it is still not sharp enough to capture all the clinical data shown. This result thus suggests that there is a different saturation dynamic physiologically than is captured (fully) by Michaelis-Menten terms, at least over this range of clinical studies.

Interestingly, the ND and RM model have qualitatively very similar errors. Thus, comparing Figures 5-7 to Figures 9-10, greater similarity might have been expected. This result shows how this PD surface approach can clearly delineate model differences more than point-wise error values. More specifically, it shows dual Michaelis-Menten saturations provide a better fit to otherwise variable clinical glycaemic clamp data than a single saturation, but that the shape of these saturation curves may not be ideal, at least in this case.

However, the MM model is distinctly different, trying to adapt to its lack of dynamics by a lower $S_{I}$ value at the lower limit, matching reported under prediction when used in clamp studies. Importantly, the similar $S_{I}$ value for ND1 results in much smaller errors and is due to a trade off between $S_{I}$ and $\alpha_{G 2}$. The MM model $S_{I}$ must be lowered by $10 \mathrm{x}$ to get RMS errors less than 0.5 or close to ND1 results, showing the reported under prediction of $S_{I}$ outside a physiological range for this model. These results also show how this PD surface analysis can clearly differentiate models.

For further illustration, Figures 12-14 show the 3D PD surfaces for the RM, ND1 and ND2 models. They clearly show the flattening effect of the individual saturation dynamics, as well as the sharper saturation from Equation (7). However, qualitatively the fit to clinical data appears similar.

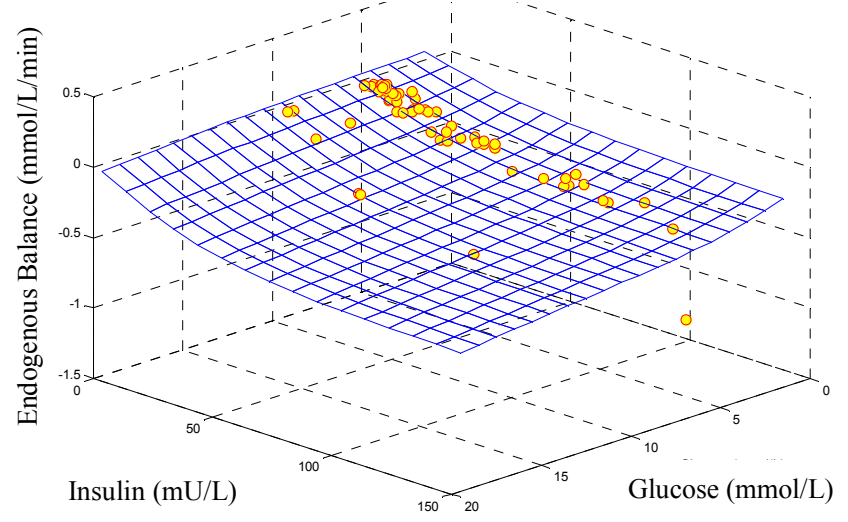

Figure 12: RM 3D surface, dots are clinical studies

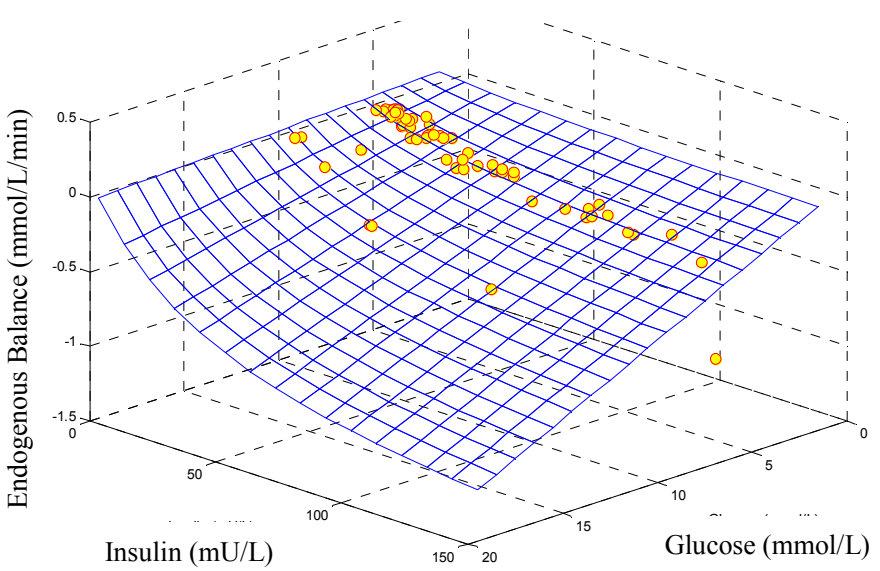

Figure 13: ND1 3D surface, dots are clinical studies

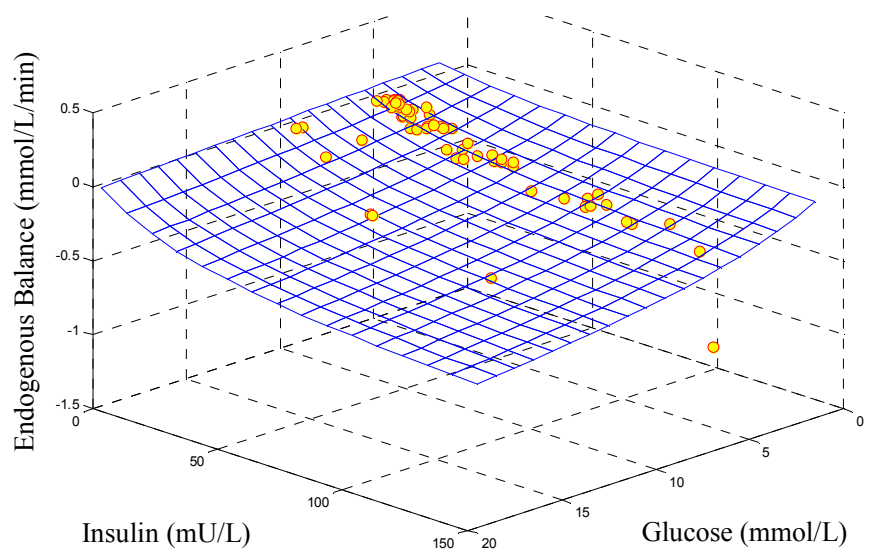

Figure 14: ND2 3D surface, dots are clinical studies 
In clinical situations, the ND models have shown very good predictive accuracy to within measurement error over $92 \%$ of the time, validating them for their clinical use in critical care (e.g. Wong et al, 2006; Hann et al, 2005). The RM model has less clinical validation, but 1-2 hour prediction accuracy using the same data (unpublished) yield essentially equivalent results to the ND model. Hence, both models, despite differences in the surfaces and dynamics in this analysis, are clinically effective for glycaemic management.

Thus, the primary differences revolve around the shape of the saturation curve for insulin and the multiple glucose-insulin saturation dynamics. It is clear from this data that the sharper saturation of Equation (7) is potentially more physiological and should be validated with larger data sets. The separate saturations could also be incorporated into the ND model of Equation (1) and examined for improved predictive power. However, the current predictive accuracy of the ND and RM models is within measurement error over $90 \%$ of the time, so it may be difficult to further differentiate these models.

This situation does not hold true for the Minimal Model (MM). This model is clearly deficient outside of low insulin and glucose values in Figures 4-5. The 3D surface of Figure 15 with a z-axis $3 x$ larger than Figures 12-14 clearly shows this result. The lack of well known physiological saturations and effects is clearly evident here, as in Table 2.

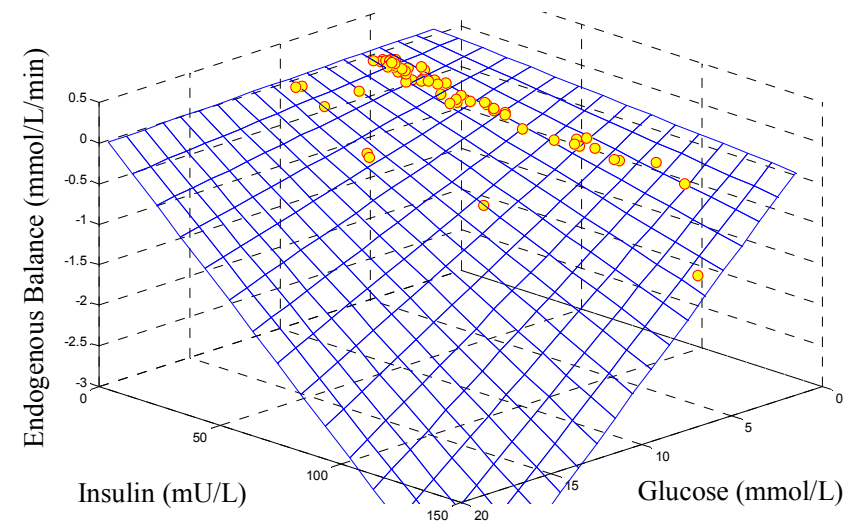

Figure 15: MM 3D surface, dots are clinical studies.

\section{CONCLUSIONS}

A new PD surface oriented approach to model validation has been presented for metabolic systems models. Two nonlinear, physiologically representative dynamic models (ND) a receptor-based (RM) model and the well-known Minimal Model (MM) were examined. The ND and RM models provide similar dynamics with salient differences due to the exact method of adding physiological saturation dynamics. In contrast, the $\mathrm{MM}$ is not physiologically representative enough to provide a $3 \mathrm{D}$ surface that captures the clinical data outside low insulin and glucose ranges, illustrating the difficulty in using this model for predictive glycaemic control. The reported under-prediction of insulin sensitivity by the MM is clearly evident. Comparing ND1 and ND2 shows the need for two saturation terms in the ND1 model due to trade offs between insulin sensitivity and the second saturation term. The RM model would benefit from validation on additional data in future work.

\section{REFERENCES}

Arleth, T., S. Andreassen, M.O. Federici, and M.M. Benedetti, A model of the endogenous glucose balance incorporating the characteristics of glucose transporters, Comput Methods Programs Biomed, 2000, 62(3), pp. 219-34

Chase, JG, Hann, CE, Shaw, GM, Wong, XW, Lin, J, Lotz, T, LeCompte, A and Lonergan, T (2007). "An Overview of Glycemic Control in Critical Care - Relating Performance and Clinical Results," Diabetes Science and Technology, Vol 1(1), pp. 82-91

Egi M, Bellomo R, Stachowski E, French CJ, Hart G: Variability of blood glucose concentration and short-term mortality in critically ill patients. Anesthesiology 2006, 105(2), pp. 244-252

Hann, C.E., J.G. Chase, J. Lin, T. Lotz, C.V. Doran, and G.M. Shaw, Integral-based parameter identification for longterm dynamic verification of a glucose-insulin system model, Comput Methods Programs Biomed, 2005, 77(3), pp. 259-70

Hovorka, R., V. Canonico, L.J. Chassin, U. Haueter, M. Massi-Benedetti, M.O. Federici, T.R. Pieber, H.C. Schaller, L. Schaupp, T. Vering, and M.E. Wilinska, Nonlinear model predictive control of glucose concentration in subjects with type 1 diabetes, Phys Measmt, 2004, 25(4), pp. 905-920

Krinsley JS (2004) Effect of an intensive glucose management protocol on the mortality of critically ill adult patients. Mayo Clin Proc 79, pp. 992-1000

Krinsley JS, Jones RL (2006) Cost analysis of intensive glycemic control in critically ill adult patients. Chest 129, pp. 644-650

Lotz, T, Chase, JG, McAuley, KA, Lee, DS, Lin, J, Hann, CE and Mann, JI (2006). "Transient and steady state euglycemic clamp validation of a model for glycemic control \& insulin sensitivity testing," Diabetes Technology \& Therapeutics, Vol 8(3), pp. 338-346

Pacini, G. and R.N. Bergman, MINMOD: a computer program to calculate insulin sensitivity and pancreatic responsivity from the frequently sampled intravenous glucose tolerance test, Comput Methods Programs Biomed, 1986, 23(2), pp. 113-22

Van den Berghe G, Wouters P, Weekers F, et al. (2001) Intensive insulin therapy in the critically ill patients. $\mathrm{N}$ Engl J Med 345, pp. 1359-1367

Wong, XW, Shaw, GM, Hann, CE, Lotz, T, Lin, J, SinghLevett, I, Hollingsworth, L, Wong, OS and Chase, JG (2006). "A novel, model-based insulin and nutrition delivery controller for glycemic regulation in critically ill patients," Diabetes Technology \& Therapeutics, Vol 8(2), pp. 174-190 